\title{
L'ingénierie lexicale ou la description d'un objet entre l'invention et la découverte*
}

\author{
Jean-Nicolas De Surmont, Université de Leeds, Leeds, Royaume-Uni \\ (jdesurmont@yahoo.fr)
}

\begin{abstract}
Résumé: Résoudre des problèmes théoriques constitue également résoudre des problèmes lexicaux. C'est ce que nous avons baptisé l'ingénierie lexicale, processus d'évaluation et de description d'une activité ou d'une discipline et de création de termes ou de lexies pouvant satisfaire les besoins théoriques du chercheur. La nécessité de recourir à l'ingénierie lexicale peut apparaître dans différents contextes scientifiques notamment les études transdisciplinaires, les études diachroniques, l'harmonisation interlinguistique, etc. Nous présentons donc différents cas de figures notamment dans le cadre de nos travaux sur la poésie vocale.
\end{abstract}

Mots-clés: INGÉNIERIE LEXICALE, RÉSOLUTION DE PROBLÈME (LEXIQUE), LEXICOLOGIE, TERMINOLOGIE, ÉPISTÉMOLOGIE

\begin{abstract}
Lexical Engineering or the Description of an Object between Invention and Discovery. Solving theoretical problems also involves solving lexical problems. This we have named lexical engineering, a process of evaluation and description of an activity or a discipline and the creation of terms or lexical items, which have to satisfy the theoretical needs of the researcher. The necessity to resort to lexical engineering becomes apparent in different scientific contexts, notably transdisciplinary studies, diachronic studies, interlinguistic harmonization, etc. We therefore present different kinds of figurative senses notably as part of our work on vocal poetry.
\end{abstract}

Keywords: LEXICAL ENGINEERING, SOLUTION OF (LEXICAL) PROBLEMS, LEXICOLOGY, TERMINOLOGY, EPISTEMOLOGY

\section{L'ingénierie lexicale: définition}

Loin de nous l'idée de défendre une quelconque hypothèse nominaliste ou réaliste du langage semblable aux célèbres thèses soutenues par Cratyle et Hermogène dans Platon si ce n'est que de montrer que l'ingénierie lexicale

* Ce texte constitue la version écrite d'une présentation ayant eu lieue lors d'une journée d'études organisée à Cergy-Pontoise intitulée «La lexicographie, rayonnement d'hier à demain et lexiculture» le 20 novembre 2009. Des éléments présentés ici reprennent les idées développées dans l'ouvrage Chanson: son histoire et sa famille dans les dictionnaires de langues française, Berlin/New York, Walter de Gruyter, 2010. 
s'inscrit dans une démarche intellectuelle faite de conventions et de vide lexical (syntagme que j'emploie par analogie à vide juridique). L'ingénierie lexicale répond aux besoins des linguistes et théoriciens des sciences humaines qui créent des dénominations, étudient le sens en contextes ou les significations de dénominations préexistantes. Ainsi à partir d'une étude sur la définition prototypique de grève dans les dictionnaires courants actuels, Tournier (2002: 277) en vient à la conclusion suivante:

Seule une définition prototypique, établie à partir de réponses faites à un questionnaire sociolinguistique, serait vraiment synchroniste, donc structuraliste. Une véritable enquête interrogeant un panel représentatif de la population française adulte est seule légitimée à apporter ces réponses. Elle ferait peut-être apparaître qu'aujourd'hui la grève est ressentie comme un phénomène plus général et moins spécialisé qu'autrefois. Elle montrerait sans doute aussi le flou des significations qui préside à l'usage des mots.

Voilà résumé les problèmes auxquels Tournier a été confronté en faisant une analyse diachronique de certains lexèmes du champ sémantique et des cooccurents de grève.

Devant une telle situation que ce soit l'analyse d'un phénomène social ou d'une discipline, le théoricien bénéficie souvent du travail du métalexicographe comme le montrent les nombreux ouvrages d'introduction ou synthétique en sciences humaines, commençant par une définition très succincte de la discipline étudiée, définition souvent extraite, en langue française, du Petit Larousse ou du Petit Robert. Mais il faut constater les limites de ces définitions lorsque le théoricien en vient aux prises à des problèmes similaires à ceux que soulève Tournier. Pour cette raison j'ai créé le terme «ingénierie lexicale», et expliqué l'activité de résolution de problèmes théoriques. Ainsi, l'activité néologique propre à l'ingénierie lexicale constitue un ensemble de prises de décisions cherchant à répondre aux problèmes soulevés lors de l'enquête terminologique en diachronie.

\section{Ingénierie terminologique}

Dans la première partie de notre texte nous avons évoqué l'utilité de l'ingénierie lexicale afin de proposer des solutions aux problèmes rencontrés dans le cadre d'une activité reliée à l'usage des vocabulaires de spécialité en diachronie. La création d'un vocabulaire supradisciplinaire constitue l'un des objectifs de l'ingénierie terminologique. Dans ce cadre, elle peut s'avérer une solution socioterminologique en satisfaisant les impératifs nominatifs et conceptuels d'une communauté linguistique et culturelle. En partant du concept d'ingénierie lexicale j'en ai déduit celui d'ingénierie terminologique. L'ingénierie terminologique consiste à étudier globalement un champ disciplinaire afin d'y proposer des solutions empiriques, par exemple la création néologique ou la création d'archi-concepts opératoires. Sur le plan cognitif, l'ingénierie terminologi- 
que se décline en un va-et-vient épistémologique, une négociation sémantique qui va de l'onomasiologie à la sémasiologie, de l'induction et de la déduction. Traugott (1998: folio 5) affirme à ce sujet: «Ultimately of course, semasiology presupposes onomasiology, and onomasiology presupposes semasiology, since domains have members, and over times individual form-meaning pairs come to be used to express those domains [...].» Traditionnellement l'activité du terminologue consiste à l'élaboration du fichier de dépouillement par le repérage des unités terminologiques ce qui constitue en quelque sorte une activité de cartographie pré-sémasiologique d'un champ disciplinaire. Les données métaterminologiques sont extraites des monographies, dictionnaires de spécialités et servent à la réflexion sémasiologique lors de l'enquête terminologique.

Même si en apparence, la résolution d'un problème de dénomination cherche la simplification, l'ingénieur terminologique cherche plutôt à juger des imperfections théoriques, selon le concept de Shapere (1977), qui se manifestent entre autres, lorsque la théorie proposée se révèle incomplète relativement au domaine visé, ou ne constitue qu'une simplification. Les procédés de traitement des néonymes ont été largement commentés dans les travaux de terminologues comme Loïc Depecker, Teresa Cabré, Daniel Gouadec: les emprunts directs: emprunt intégral (importation de la forme et du sens sans modification significative), emprunt sémantique, emprunt aménagé (aménagement de la forme, et éventuellement du sens), les emprunts par traduction: calque (traduction littérale du terme étranger), calque transpositionnel (transposition non littérale), synthèse néologique (reconceptualisation) (voir Depecker 2001: 403), inversion syntaxique des éléments de la langue anglaise, etc.

Parmi les imperfections théoriques qui sont notamment liés à l'étude d'un champ disciplinaire en diachronie mentionnons la synonymie. Ainsi, le phénomène de synonymie appelle celui de coexistence terminologique, que nous avons créé pour désigner le fait qu'un signifié identique est nommé par des dénominations différentes selon les perspectives d'études et les périodes ou encore que des référents différents sont lexicalisés de manière similaire en un même moment donné. Les dénominations, bien que différentes, ont des sens apparentés mais non équivalents. Il s'agit donc d'un concept proche de celui de synonymie propre à la sémantique et de terme associé employé en indexation ${ }^{1}$ mais différent en cela qu'il ajoute une dimension diachronique au phénomène de similitude sémique de même que l'idée de point de vue dénominatif divergent. Chansonnier et auteur-compositeur-interprète peuvent être considérés comme des phénomènes de coexistence terminologique. Ce concept désigne aussi le fait que des unités sont parfois en exclusion dans l'un de leurs sens mais sont synonymes dans l'autre.

\section{Problèmes à résoudre dans le cadre de l'ingénierie lexicale}

Les différents points que nous avons traités plus tôt nous permettent de soulever les problèmes inhérents à l'étude du lexique d'une discipline en diachronie: 
(1) Evolution historique des signifiés, des définitions

(2) Co-existence lexicale

(3) Hétérogénéité des activités d'un vocabuliste: activité lexicale, terminologique, mots concrets, sociaux.

(4) Absence de dénominations pour décrire des phénomènes de l'activité ou de la discipline étudiée;

(5) Etudes d'un objet multidisciplinaire: l'exemple de la chanson nécessite le recours à des archi-concepts.

Analyser l'évolution historique d'une activité sociale permet de comprendre les lacunes de la description lexicographique du lexique en mettant en perspective l'étude philologique des formes et des significations. Mais outre le flou des significations que l'auteur évoque avec raison, c'est aussi l'absence de dénominations de certains phénomènes qui devraient nous intéresser en tant qu'ingénieurs lexicaux. Les unités lexicales désignant des choses concrètes n'ont évidemment pas la même fluctuation que les dénominations de faits sociaux, esthétiques et sont sûrement plus proches dans leur fonctionnement logicosémantique des termes que des lexèmes. C'est en somme là un ensemble de problèmes auxquels nous sommes confrontés en étudiant un champ lexical ou sémantique en diachronie.

Afin de résoudre les problèmes qui se présentent dans l'étude d'une activité, d'un champ scientifique, d'une discipline, nous proposons la cartographie sémantique: il faut d'abord constituer un corpus représentatif des items lexicaux à étudier. En somme il faut étudier le vocabulaire d'un champ disciplinaire au sein d'un corpus textuel important non pas tant pour réaliser à la manière de Tournier un lexicogramme, que de dégager un réseau lexical pour cerner les vides lexicaux.

(1) Relever des imperfections théoriques par l'étude onomasiologique et sémasiologique, le repérage des coexistents lexicaux.

(2) Procéder à un éventuel recyclage terminologique par la néonymie ou la néologie.

Les premières étapes de l'étude concernent d'une part l'étude du sens (en contexte) et de la signification (hors contexte) et d'autre part la contribution néologique par la formulation d'un lexique supradisciplinaire selon le néologisme construit par Wijnands (1997: 139) dans la mesure «où il comprend un vocabulaire qui s'ajoute aux vocabulaires de chacune des disciplines participantes». Cette approche cherche à favoriser l'univocité référentielle tout en restant conscient des limites pragmatiques de l'objet d'étude et sans pour autant adopter l'univocité entre le terme et le concept propre à la pensée logique émanant de la terminologie. 


\section{Le problème de la référence dans le vocabulaire chansonnier: un flou incontournable?}

Il faut être conscient des limites que pose la création néologique dans le champ des études littéraires. En s'intéressant à des objets-chansons caractérisés par (selon Arrivé, Gadet en Galmiche 1986 cité par Beaumont-James 1994: 10) un «système de significations qui se manifestent simultanément par des signifiants de divers types» (rythme, mélodie, parole, image, voix, accompagnements, bruits), par des procédés de diffusion divers, le caractère subjectif est d'autant plus flagrant qu'il ne s'agit là que de constructions conceptuelles qui, en ce sens, évoquent le processus de formation des concepts propres aux sciences humaines et sociales. Si les faits historiques et sociaux considérés en pure synchronie sont immuables, en revanche certains aspects impliqués dans l'étude d'un vocabulaire, même en synchronie, ne le sont pas. Ainsi le métalangage, les conditions d'étude de ces objets, donc l'aspect épistémologique et sémiotique des phénomènes chansonniers, vont influer sur la description lexicographique des unités lexicales de la famille morphologique de chanson. C'est aussi la littérarité d'une forme aussi controversée que la chanson qui est sujette aux changements. Budin (1991: 338) précise à cet effet: «Since most of these objects described above constantly change (especially in sociology, political science etc., but certainly not in historical disciplines), empirical and - as a consequence - also theoretical concepts are modified in order to represent these objects adequately. Therefore, a certain degree of inherent fuzziness cannot be prevented». ${ }^{2}$

\section{Néologie lexicale du phénomène chansonnier}

Afin d'illustrer les propos de Budin, restons dans le cadre de la poésie vocale. Au XIXe siècle, on n'assimilait pas forcément chanson et performance vocale. Pour cette raison, l'emploi du syntagme poème chanté ou poème mis en musique et poème mis en chanson a été adopté. La présence du substantif poésie sert ici à mentionner qu'il s'agit d'un texte. Poème chanté ou encore poésie chantée rappelle donc que la vocalisation reste virtuelle d'une part et que, d'autre part, il s'agit d'un texte à auteur connu que l'on met ensuite en musique. Boiste (1834) l'exprime explicitement à l'entrée «chanson»: «Pièce de vers par couplets, que l'on peut chanter». Comme cette dénomination recouvre un ensemble de phénomènes de diffusion d'objet-chansons relativement hétéroclite, on a préféré chanson signée sur timbre, s'il s'agit d'une mélodie de la tradition orale, d'un poème mis en musique, ou s'il s'agit d'un compositeur qui met en musique le texte d'un poète.

Cette dimension aléatoire de la vocalisation de la chanson correspond aussi au sémème /recueil de poésie/ acception généralement consignée dans les dictionnaires de langues générale au XIXe siècle. Ces observations sur la nature fluctuante des sémèmes et forcément des objets esthétiques nous font adopter les vues de Traugott (1997: 7) pour qui la subjectivation est un phéno- 
mène diachronique: «It may result synchronically in layerings of less or more subjective meanings of the same lexical item or construction, but there is no necessary isomorphism between the diachronic and synchronic variation». ${ }^{3}$ Les données sont linguistiquement hautement construites, mentionne-t-elle, et peuvent voir des sens anciens et nouveaux coexister synchroniquement.

Cette approche conceptologique met en valeur le caractère subjectif de la stabilité des concepts mais aussi de leur élaboration. Elle fait bien voir en outre que l'idéal lexicographique d'une représentation mentale abstraite et générale n'échappe pas au contexte institutionnel et culturel dans lequel il est élaboré.

L'ensemble des procédés d'ingénierie terminologique vise à restaurer la puissance polysémique, à tenir compte de la variation diachronique et à étudier les éventuelles absences de dénominations dans un champ terminologique. L'ingénierie terminologique et lexicale est un procédé métaterminologique traduisant les différents processus impliqués dans la création lexicale et dans le cycle de vie terminologique, que ce soit la néologisation ou la normalisation (ou de normaison, concept que nous devons à Guespin 1975). L'ingénierie terminologique consiste donc à proposer des éléments de solutions en réponse au vide terminologique et aux ambiguïtés pragmatiques liées aux changements de sens en diachronie et à la variabilité des contenus conceptuels sur le plan diachronique (ainsi l'acquis de l'Union (Traité de Nice) inclut l'acquis communautaire (Traité d'Amsterdam) ). L'existence du syntagme seconde génération pour évoquer les enfants d'immigrants traduit la nécessité historique de la distinguer de la génération précédente. Il s'agit là d'un exemple d'ingénierie lexicale parmi tant d'autres dont la valeur terminologique pourrait aussi être contesté bien que le terme figure dans certains dictionnaires spécialisés (Bolaffi et al. 2003: 199). L'ingénierie terminologique s'intéresse en ce sens à la valorisation sociale de certains usages au détriment de certains autres. Elle peut être le fait d'un essayiste qui crée son vocabulaire opératoire comme elle peut être le fait d'organismes officiels qui proposent, recommandent des néonymes à une communauté intellectuelle.

\section{L'usage d'un vocabulaire empirique}

Dans le cadre de mes travaux de recherches depuis dix ans j'ai notamment divisé l'objet-chanson en deux grandes familles (la chanson de tradition orale et la chanson signée) et j'ai aussi introduit quelques dénominations qui répondent à la difficulté de cerner ce qu'est une «chanson» de ce qui n'en est pas une. Si l'on considère avec Wittgenstein ([1922], 1966: 29) que «le monde est l'ensemble des faits, non pas des choses», nous déduisons celui de fait chansonnier qu'utilise Marcadet (1999) sans doute inspiré du concept de Green (1998) issu de l'anthropologie française fait musical. Nous préférons l'emploi de phénomène chansonnier à cette première dénomination qui nous semble objectiviste, voire positiviste. D'abord comment cerner les contours conceptuels d'un genre? Dans la mesure où le mot chanson regroupe des esthétiques ${ }^{4}$ des objets-chansons 
divers en plus d'être étudié selon de multiples points de vue, il convient en ce sens d'utiliser phénomène chansonnier. Le phénomène chansonnier signifie l'ensemble des pratiques, manifestations ou traditions, et supports diffusant et médiatisant l'objet-chanson, d'une part, et, d'autre part, l'ensemble des recherches et publications sur la chanson ou qui influencent son statut dans les champs culturel, économique et symbolique. Cela inclut toutes les monographies ou articles concernant la chanson (biographie, étude, essais, etc.). Décrire un phénomène chansonnier, c'est donc parler d'une multitude d'objets-chansons possédant certaines caractéristiques communes sur le plan du processus créatif et des canaux de diffusion (Wierzbicka 1985: 191). ${ }^{5}$ Le concept de phénomène chansonnier renvoie donc plus à un construit social, historique et historiographique qu'à l'objet chanson lui-même.

Si j'ai préféré phénomène chansonnier au concept déjà existant de fait chansonnier (voir entre autres Marcadet 1999: 291), qui renvoie à l'origine des chansons, aux performances et aux impacts qu'elles suscitent, c'est à cause d'une vision propre à la théorie de la connaissance qui fait du phénomène, au sens kantien du terme, non une chose en soi mais une donnée de l'expérience. En ce sens, relève de chanson ce qui est considéré comme tel par la conscience. Dans ce cas il devient objet-chanson. Par objet-chanson, j'entends le résultat final du procédé d'interprétation du phénomène chansonnier et l'objet généralement reconnu comme une chanson (celle décrite par le sémème: / pièce de vers chanté sur un air/), bref l'objet de la performance. L'objet-chanson constitue un sous-ensemble des phénomènes chansonniers et sert à alimenter l'activité chansonnière, laquelle est tout aussi bien relative à l'activité de publication de recueils, qui a retenu l'intérêt des médiévistes, qu'à celle des médiateurs qui «colportent» des chansons, laquelle a surtout retenu l'attention des folkloristes.

\section{Recyclage terminologique}

J'ai évoqué plus tôt dans les solutions que propose l'ingénieur aux imperfections théoriques le recyclage terminologique. Contrairement au marquage en lexicologie qui fait cohabiter les marques d'usages obsolètes ou anciens et les marques de nouveautés, de celui des topolectismes où prédomine le marquage des usages archaïques au détriment des usages nouveaux, celui des termes revêt plutôt le caractère d'institutionnalisation du signe linguistique (selon la théorie d'Alinei 1997) et diverge du parcours de la consécration lexicographique dont le point de départ méthodologique, du moins en théorie, est le texte. En observant les trois phases du cycle de vie terminologique (sa naissance, sa vie et sa mort), on prend conscience de la vitalité d'un emploi. Les pratiques lexicologiques et terminologiques diffèrent sur ce point. On peut en effet remarquer que le fait de consigner une unité relève d'un choix dans la description d'un certain usage (lexicologie) ou d'une stratégie d'implantation d'un terme récemment proposé par un organisme officiel (terminologie). La trajectoire métalinguistique se prête ainsi à une lecture thématique, organisée sur un 
champ lexical et sémantique comportant un effort d'historicisation, retraçant le cycle de vie des termes et des notions (voir Collinot et Mazière 1997: 1). Ce sont là des preuves de la vitalité d'un mot puisque l'inscription d'un lemme dans la nomenclature pour la première fois passe d'un usage réduit, dans l'absolu idiolectal, à un signe public (ou plutôt publicisé). L'étape ultime de changement consiste à adopter un néologisme et pourrait se nommer riciclaggio dei segni preesistenti suivant le concept d'Alinei (1997: 16). Ce «recyclage terminologique» peut être notamment le fait d'une action d'un médiateur métaterminologique (comme les commissions de terminologie) qui exercent, depuis les années 1970, le rôle de critique vis-à-vis des emplois en vue de suggérer des solutions néonymiques pour la description d'un champ d'activité supradisciplinaire.

\section{Conclusion}

La théorie générale de la terminologie et les contraintes de la mouvance référentielle sont l'objet de cet article.

Bien qu'il ait été ici procédé pour une partie de notre exposé à un travail d'onomasiologue où normalement les concepts sont nécessairement construits en vue d'édifier un arbre conceptuel, un champ notionnel bien délimité, on montre quelques réserves envers ce modèle traditionnel logico-déductif de formation des concepts. ${ }^{6}$ Nous sommes d'avis que l'étude d'un vocabulaire d'une part, et des phénomènes chansonniers d'autre part, implique des procédés d'abstraction qui ne s'excluent pas l'un l'autre. Le vocabulaire se place du côté du signifiant, les phénomènes chansonniers du côté du référent et les significations du côté des constructions conceptuelles.

Le processus d'ingénierie lexicale induit par celui d'un lexique explicatif supradisciplinaire nous a fait percevoir que le flou du vocabulaire chansonnier n'est pas uniquement dû à une méconnaissance des processus de composition, des registres, des caractéristiques profondes qui permettent une meilleure identification des genres chansonniers, mais aussi et surtout à ce que l'objetchanson est un objet bâtard.

En restant conscient de cette nature fluctuante, éphémère des concepts et dénominations propres aux sciences humaines, nous avons proposé un lexique explicatif. Cette approche conceptologique met en valeur le caractère subjectif de la stabilité des concepts mais aussi de leur élaboration. Elle fait bien voir en outre que l'idéal lexicographique d'une représentation mentale abstraite et générale n'échappe pas au contexte institutionnel et culturel dans lequel il est élaboré. Il n'échappe pas non plus aux limites des corpus aussi bien dans la tradition d'érudition des lexicographes traditionnels que dans les corpus électroniques, qui, en langue française du moins, sont encore peu représentatifs de la fréquence d'usage de certaines unités complexes comme chanson populaire.

Dans la mesure où nous considérons globalement l'ingénie lexicale comme la science de régulation, de recyclage des métatermes mais aussi plus généralement la science de la résolution des problèmes lexicaux et terminologique, 
rien ne nous empêche de considérer certaines pratiques d'auteurs au sein de l'ingénierie lexicale, comme c'est le cas d'Hubert Aquin chez qui l'ont trouve des lexiques qui sont des dossiers préparatoires ayant servi à l'élaboration de certains romans, certains étant, selon l'expression même de l'auteurs des «mots générateurs» (Dupuis 2005: 49).

\section{Notes}

1. Nous renvoyons à la terminologie du guide RAMEAU (Répertoire d'Autorité-Matière Encyclopédique et Alphabétique Unifié 1995).

2. Traduction libre: «Dès lors que ces objets décrits [...] changent constamment (spécialement en sociologie, science politique, etc. mais certainement pas dans les disciplines historiques) les concepts empiriques, et par conséquent théoriques, sont modifiés dans le but de représenter ces objets adéquatement. Un certain degré de flou intrinsèque ne peut être alors évité».

3. Traduction libre: «Il peut résulter synchroniquement dans la superposition d'un ou de plusieurs sens subjectifs de la même unité ou construction lexicale, mais il n'y a pas d'isomorphisme entre la variation diachronique et synchronique».

4. Notons les affirmations critiquées de Theodor Adorno en ce domaine parce qu'il prétendait que les caractéristiques esthétiques de la chanson de masse constituaient la moyenne, ni plus ni moins, de l'ensemble des chansons présentées sur le marché. La chanson, pour se vendre, doit faire l'objet d'un rapport avec les goûts de la masse et devenir ainsi un objet de consommation soumis aux mêmes conditions de mises en marché que les biens capitalistes.

5. Il existe tout autant d'objets-chansons que d'occurrences de phénomènes chansonniers activant la facette /pièce de vers/. Je ne considère pas comme objet-chanson les références de /parole/, /rengaine/ qui sont les sens figurés (voir Du Chazaud 1991, s. v. chanson). Pour une liste exhaustive de synonymes, on se reportera au dictionnaire signé par l'équipe de Sabine Ploux, Jean-Luc Manguin, et Bernard Victorri qui ont constitué une base de départ de sept dictionnaires de langue française (Bailly, Bénac, Du Chazaud, Guizot, Lafaye, Larousse et Robert). Il donne 62 synonymes de chanson. Disponible à l'adresse http://elsap1.unicaen. fr/dicosyn.html. Juin 2003.

6. Notre réflexion est conduite en partie par l'ouvrage de Bolton (1977).

\section{Bibliographie}

Alinei, Mario. 1997. Principi di teoria motivazionale (iconimia) e di lessicologia, motivazionale (iconomastica). Mucciante, Luisa et Tullio Telmon (Éds.). Lessicologia e Lessicografia. Atti del Convegno della Società Italiana di Glottologia. Chieti-Pescara 12-14 ottobre 1995: 9-36. Rome: Il Calamo.

Arrivé, Michel, Françoise Gadet et Michel Galmiche. 1986. La grammaire d'aujourd'hui. Paris: Flammarion.

Beaumont-James, Colette. 1995. Analyse sémantique du mot chanson. Cahiers de lexicologie 67(2): 163-192.

Bolton, Neil. 1977. Concept Formation. Oxford: Pergamon.

Budin, Gerhard. 1988. The Application of Terminology-based Knowledge Data Bases in the Humanities and the Social Sciences and Its Impact on Research Methods. Best, Heinrich, 
Ekkehard Mochmann et Manfred Thaller (Éds.). 1991. Computers in the Humanities and the Social Sciences: 337-342. München/Londres/New York/Paris: Saur.

Collinot, André et Francine Mazière. 1997. Un prêt à parler: le dictionnaire. Paris: PUF.

Depecker, Loïc. 2001. L'invention de la langue: Le choix des mots nouveaux. Paris: Armand Colin, Larousse.

De Surmont, Jean-Nicolas. 2010. Chanson: son histoire et sa famille dans les dictionnaires de langue française. Berlin/New York: Walter de Gruyter.

De Surmont, Jean-Nicolas. 2005. L'ingénierie terminologique comme contribution à la constitution d'un vocabulaire supradisciplinaire. Le langage et l'homme 40(1): 123-136.

Dupuis, Gilles. 2005. Du mot juste au mot rare. Dictionnaires et glossaires dans l'œuvre d'Hubert Aquin et Jacques Poulin. Acerenza, Gerardo (Dir.). 2005. Dictionnaires français et littératures québécoise et canadienne-française: 35-50. Ottawa: Éditions David.

Green, Anne-Marie. 1998. Musicien de métro. Approches vivantes urbaines. Paris/Montréal: L'Harmattan.

Guespin, Louis. 1975. Normaliser ou standardiser? Le langage et l'homme 28(4): 213-222.

Guespin, Louis. 1985. Matériaux pour une glottopolitique. Problèmes de glottopolitique, Cahiers de Linguistique Sociale 7: 13-32.

Marcadet, Christian. 1999. La matrice des faits-chansons: le cas Yves Montand. Quéniart, Jean (sous la direction de). 1999. Le Chant, acteur de l'histoire: 291-306. Rennes: Presses Universitaires de Rennes.

Shapere, Dudley. 1977. Scientific Theories and their Domains. Suppe, Frederick (Éd.). 1977. The Structure of Scientific Theories: 518-599. Deuxième édition. Urbana: University of Illinois Press.

Tournier, Maurice. 2002. Des mots sur la grève. Propos d'étymologie sociale 1. Lyon: ENS Éditions.

Traugott, Elizabeth Closs. 1997. The Rhetoric of Counter-expectation in Semantic Change: A Study in Subjectification. Blank, Andreas et Peter Koch (Éds.). 1999. Historical Semantics and Cognition: 177-196. Berlin/New York: Mouton de Gruyter.

Wierzbicka, Anna. 1985. Lexicography and Conceptual Analysis. Ann Arbor: Karoma Publishers.

Wijnands, Paul. 1997. L'interdisciplinarité du discours sur l'identité culturelle: les mots d'auteur. De Schaetzen, C. (Éd.). 1997. Terminologie et interdisciplinarité. Actes du colloque organisé en avril 1996 par le Centre de terminologie de Bruxelles (Institut Libre Marie Haps) et l'Association européenne des Professeurs de Langue vivantes (AEPLV): 137-146. Louvain-la-Neuve: Peeters.

Wittgenstein, Ludwig. 1961. Tractatus logico-philosophicus, suivi des investigations philosophiques. Paris: Gallimard. (L'édition d'orgine est celle de Londres: Kegan Paul, 1922.)

\section{Dictionnaires}

Boiste, Pierre Claude Victoire. 1834. Dictionnaire universel de la langue française, avec le latin et les étymologies [...]. Huitième édition [revue par C. Nodier]. Paris: Lecointe et Pougin, Quai des Augustines. S.v. «Chanson».

Bolaffi, Guido, Raffaele Bracalente, Peter Braham et Sandro Gindro. 2003. Dictionary of Race, Ethnicity \& Culture. Londres: Sage Publications.

Du Chazaud, Henri Bertaud. 1991. Dictionnaire de synonymes et contraires. Paris: Le Robert.

[En Coll.]. 1999. [PLI] Le petit Larousse illustré. Paris: Larousse.

Rey-Debove, Josette et Alain Rey (texte remanié et amplifié sous la dir. de). 1993. [NPR1] Le Nouveau Petit Robert, dictionnaire alphabétique et analogique de la langue française. Paris: Dictionnaires Le Robert. 